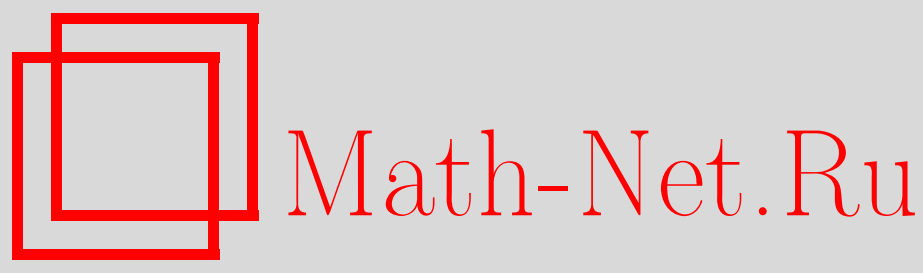

С. В. Захаров, Асимптотическое решение многомерного уравнения Бюргерса вблизи сингулярности, ТМФ, 2018, том 196, номер 1, 42-49

DOI: https://doi.org/10.4213/tmf9479

Использование Общероссийского математического портала Math-Net.Ru подразумевает, что вы прочитали и согласны с пользовательским соглашением http://www.mathnet.ru/rus/agreement

Параметры загрузки:

IP : 3.95.254.165

26 апреля 2023 г., 13:07:55

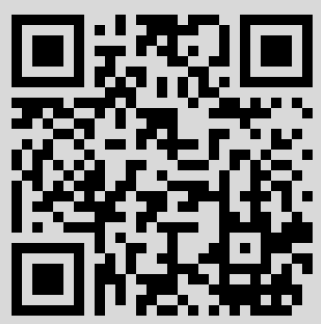




\section{АСИМПТОТИЧЕСКОЕ РЕШЕНИЕ МНОГОМЕРНОГО УРАВНЕНИЯ БЮРГЕРСА ВБЛИЗИ СИНГУЛЯРНОСТИ}

\footnotetext{
Рассматривается задача Коши для многомерного уравнения Бюргерса с малым параметром диссипации. Методом согласования строится асимптотическое решение вблизи сингулярности, обусловленной структурой векторного поля в начальный момент времени. Использованный в работе подход позволил проследить эволюцию решения с иерархией разномасштабных структур и дать строгое математическое определение асимптотического решения в главном приближении. Обсуждается связь рассматриваемой задачи с различными моделями фундаментальной и прикладной физики.
}

Ключевые слова: многомерное уравнение Бюргерса, задача Коши, асимптотика.

DOI: https://doi.org/10.4213/tmf9479

\section{1. ВВЕДЕНИЕ}

В настоящей работе рассматривается задача Коши для многомерного уравнения Бюргерса

$$
\begin{gathered}
\frac{\partial \mathbf{v}}{\partial t}+(\mathbf{v} \nabla) \mathbf{v}=\varepsilon \triangle \mathbf{v}, \quad t \geqslant 0 \\
\mathbf{v}(x, 0, \varepsilon, \mu)=\mu \nabla \Lambda\left(\frac{x_{1}}{\mu}, \ldots, \frac{x_{m}}{\mu}\right),
\end{gathered}
$$

где $x=\left(x_{1}, \ldots, x_{m}\right) \in \mathbb{R}^{m}, m \geqslant 2, \mathbf{v}=\left(v_{1}, \ldots, v_{m}\right)$ - потенииальное векторное поле, $\varepsilon$ и $\mu$ - независимые малые положительные параметры, $\Lambda(s)=\Lambda\left(s_{1}, \ldots, s_{m}\right)$ - непрерывно дифференцируемая функция с ограниченными частными производными.

Уравнение (1) нашло широкое применение в механике сплошных сред [1]-[3]. Кроме того, при размерности пространства $m=3$ оно используется в космологической

Работа выполнена при поддержке комплексной программы ФНИ УрО РАН "Аналитические, асимптотические и численные методы построения решений прямых, обратных и сингулярно возмущенных задач математической физики” (проект № 0387-2016-0039).

${ }^{*}$ Институт математики и механики им. Н.Н. Красовского Уральского отделения РАН, Екатеринбург, Россия. E-mail: SVZ@imm.uran.ru 
модели крупномасштабной структуры Вселенной [4]-[6], а случай малых $\varepsilon$ непосредственно связан с возникновением наблюдаемого распределения галактик на расстояниях, исчисляемых миллиардами световых лет.

Основная цель данной работы - построить асимптотическое решение задачи (1), (2) при $\varepsilon \rightarrow+0$ и $\mu \rightarrow+0$ вблизи сингулярности, обусловленной структурой векторного поля в начальный момент времени. Несмотря на то что типы особых точек решений подробно классифицированы [7] и процессы формирования ударных волн для предельного уравнения первого порядка изучены [8], [9], аналитическое построение асимптотик решений в окрестностях особых точек уже в одномерном случае представляет собой отдельную и иногда довольно сложную задачу [10]-[12]. При этом результаты существенно зависят от соотношения параметров $\varepsilon$ и $\mu$ [13]. В настоящем исследовании считается, что

$$
\frac{\mu}{\varepsilon} \rightarrow 0
$$

Разумеется, в рассматриваемой здесь задаче асимптотику можно получить из явной формулы, которая находится с помощью преобразования Коула-Хопфа

$$
\mathbf{v}=-2 \varepsilon \frac{\nabla U}{U}, \quad \frac{\partial U}{\partial t}=\varepsilon \triangle U
$$

Несмотря на существование такой формулы для точного решения, в выражении, полученном ниже методом согласования [10], зависимость от малых параметров задачи сразу приобретает ясный смысл, а именно, становятся видны пространственно-временные масштабы, на которых происходит эволюция системы вблизи сингулярности. И хотя здесь речь идет о малых временах, подобные сингулярные события во многом определяют все последующее поведение системы, что видно уже на примере одномерного уравнения [10], [11]. Применительно к моделированию крупномасштабного распределения вещества во Вселенной это соответствует, в частности, формированию низкоразмерных структур на больших временах [4]. Что касается результата, полученного в настоящей работе, его можно использовать также в качестве примера эволюции скейлинговых (пространственно-временных) аномалий, которые появляются в различных областях физики: релятивистской гравитации [14], [15], магнетизме [16], сверхпроводимости [17].

В работе [18] было впервые установлено, что асимптотика решения в одномерном случае при выполнении условия (3) имеет нестандартную структуру вблизи особой точки. А именно, возникают две области со специфическими пространственно-временными масштабами, а не одна, как в других известных задачах для параболического уравнения.

\section{2. ЛИНЕЙНОЕ ПРИБЛИЖКННИЕ}

Поскольку начальный потенциал в условии (2) содержит малый параметр $\mu$, начало координат является в определенном смысле точкой сингулярности. Например, если существует вторая производная $\partial^{2} \Lambda(0, \ldots, 0) / \partial s_{\alpha} \partial s_{\beta} \neq 0$, то

$$
\left.\frac{\partial v_{\alpha}}{\partial x_{\beta}}\right|_{\substack{x=0, t=0}}=\frac{1}{\mu} \frac{\partial^{2} \Lambda(0, \ldots, 0)}{\partial s_{\alpha} \partial s_{\beta}} \rightarrow \infty, \quad \mu \rightarrow 0 .
$$


Для построения приближения, асимптотически удовлетворяющего начальному условию (2), естественно "растянуть" переменные $x_{\alpha}$ на величину $\mu^{-1}$. Чтобы главная часть уравнения (1) осталась при этом эволюционной, производная по $t$ должна иметь тот же порядок, что и правая часть, т.е. порядок $\varepsilon \mu^{-2}$. Исходя из этих соображений, сделаем замену переменных

$$
\sigma_{\alpha}=\frac{x_{\alpha}}{\mu}, \quad \omega=\frac{\varepsilon t}{\mu^{2}} .
$$

Тогда уравнение $(1)$ для $h_{\alpha}(\sigma, \omega, \varepsilon, \mu) \equiv v_{\alpha}(x, t, \varepsilon, \mu)$ принимает вид ${ }^{1)}$

$$
\frac{\partial h_{\alpha}}{\partial \omega}-\sum_{\beta=1}^{m} \frac{\partial^{2} h_{\alpha}}{\partial \sigma_{\beta}^{2}}=-\frac{\mu}{\varepsilon} \sum_{\beta=1}^{m} h_{\beta} \frac{\partial h_{\alpha}}{\partial \sigma_{\beta}} .
$$

Из уравнения (5), начального условия (2) и соотношения (3) получаем линейную предельную задачу

$$
\frac{\partial H_{\alpha}}{\partial \omega}=\sum_{\beta=1}^{m} \frac{\partial^{2} H_{\alpha}}{\partial \sigma_{\beta}^{2}}, \quad H_{\alpha}(\sigma, 0)=\frac{\partial \Lambda(\sigma)}{\partial \sigma_{\alpha}}
$$

с общеизвестным решением

$$
H_{\alpha}(\sigma, \omega)=\frac{1}{(4 \pi \omega)^{m / 2}} \int_{\mathbb{R}^{m}} \frac{\partial \Lambda(s)}{\partial s_{\alpha}} e^{-|\sigma-s|^{2} / 4 \omega} d s, \quad 1 \leqslant \alpha \leqslant m .
$$

Для уточнения вида сингулярности далее предполагаем, что выполнено асимптотическое условие

$$
\Lambda\left(\sigma_{1}, \ldots, \sigma_{m}\right)=\Theta\left(\sigma_{1}\right) \sum_{n=0}^{\infty} \frac{\Lambda_{n}\left(\sigma_{2}, \ldots, \sigma_{m}\right)}{\sigma_{1}^{n}}, \quad \sigma_{1} \rightarrow \infty,
$$

где $\Theta\left(\sigma_{1}\right)$ - функция Хевисайда, $\Theta\left(\sigma_{1}\right)=1$ при $\sigma_{1} \geqslant 0, \Theta\left(\sigma_{1}\right)=0$ при $\sigma_{1}<0$,

$$
\operatorname{supp} \Lambda_{n} \subset\left\{\left|\sigma_{2}\right|+\cdots+\left|\sigma_{m}\right|<R_{n}, R_{n}=\text { const }>0\right\} .
$$

Кроме того, пусть

$$
\operatorname{supp} \Lambda \subset\left\{\sigma_{1}>0,\left|\sigma_{2}\right|+\cdots+\left|\sigma_{m}\right|<\left|\sigma_{1}\right|^{\nu}, \nu=\text { const }>0\right\} .
$$

Тогда из результатов работы [19] вытекает, что при $\omega+|\sigma|^{2} \rightarrow \infty$ справедлива асимптотическая формула

$$
\frac{1}{(4 \pi \omega)^{m / 2}} \int_{\mathbb{R}^{m}} \Lambda(s) e^{-|\sigma-s|^{2} / 4 \omega} d s=\omega^{-m / 2} \sum_{n=0}^{\infty} \omega^{-n / 2}\left[\omega^{1 / 2} S_{n}(z)+\ln \omega \Pi_{n}(z) e^{-|z|^{2}}\right],
$$

где $z=\left(z_{1}, \ldots, z_{m}\right)$ - автомодельные переменные,

$$
z_{1}=\frac{\sigma_{1}}{2 \sqrt{\omega}}, \quad \ldots, \quad z_{m}=\frac{\sigma_{m}}{2 \sqrt{\omega}}
$$

1) На первый взгляд может показаться, что мы получили задачу с одним малым параметром $\mu / \varepsilon$. В действительности второй малый параметр скрыт во внутренних переменных (4), и для изучения решения, например, при конечных значениях $t$ все равно придется искать асимптотику при $\omega \rightarrow \infty$. 
$\Pi_{n}(z)$ - полиномы степени $n, S_{n}(z)$ - гладкие функции медленного роста. В частности,

$$
S_{0}(z)=\frac{2 e^{-z_{2}^{2}-\cdots-z_{m}^{2}}}{(4 \pi)^{m / 2}} \int_{-z_{1}}^{\infty} e^{-u^{2}} d u \int_{\mathbb{R}^{m-1}} \Lambda_{0}\left(s_{2}, \ldots, s_{m}\right) d s_{2} \ldots d s_{m}
$$

Отсюда для функций (7) при $2 \leqslant \alpha \leqslant m$ получаем асимптотику

$$
\begin{aligned}
H_{\alpha}(\sigma, \omega)= & \frac{2 e^{-z_{2}^{2}-\cdots-z_{m}^{2}}}{(4 \pi \omega)^{(m-1) / 2}} \int_{-z_{1}}^{\infty} e^{-u^{2}} d u \int_{\mathbb{R}^{m-1}} \frac{\partial \Lambda_{0}\left(s_{2}, \ldots, s_{m}\right)}{\partial s_{\alpha}} d s_{2} \ldots d s_{m}+ \\
& +O\left(\frac{\ln \left(\omega+|\sigma|^{2}\right)}{\left(\omega+|\sigma|^{2}\right)^{m / 2}}\right), \quad \omega+|\sigma|^{2} \rightarrow \infty
\end{aligned}
$$

Имея в виду условие (8), представляется довольно естественным считать, что

$$
\frac{\partial \Lambda\left(\sigma_{1}, \ldots, \sigma_{m}\right)}{\partial \sigma_{1}}=-\sigma_{1}^{-2} \Lambda_{1}\left(\sigma_{2}, \ldots, \sigma_{m}\right)\left[1+O\left(\sigma_{1}^{-1}\right)\right], \quad \sigma_{1} \rightarrow+\infty
$$

Тогда при $\omega+|\sigma|^{2} \rightarrow \infty$ получаем формулу

$$
\begin{aligned}
H_{1}(\sigma, \omega) & =\frac{1}{(4 \pi \omega)^{m / 2}} \int_{\mathbb{R}^{m}} \frac{\partial \Lambda(s)}{\partial s_{1}} e^{-|\sigma-s|^{2} / 4 \omega} d s= \\
& =\frac{e^{-|z|^{2}}}{(4 \pi \omega)^{m / 2}}\left[\int_{\mathbb{R}^{m}} \frac{\partial \Lambda(s)}{\partial s_{1}} d s+O\left(\frac{1}{\sqrt{\omega+|\sigma|^{2}}}\right)\right] .
\end{aligned}
$$

Итак, мы нашли формальное линейное приближение $\mathbf{v}(x, t, \varepsilon, \mu) \approx \mathbf{H}\left(x / \mu, \varepsilon t / \mu^{2}\right)$.

\section{3. АСИМПТОТИЧЕСКОЕ РЕШЕНИЕ}

Опыт исследования одномерного случая [13] показывает, что приближение, полученное в переменных (4), не дает адекватного описания поведения решения при значениях $t \gg \mu^{2} / \varepsilon$, и возникает необходимость строить приближение в дополнительном внутреннем слое. Заметим, что согласно равенствам (4) автомодельные переменные $z_{\alpha}$, определенные формулами (9), выражаются через $x, t$ и $\varepsilon$ следующим образом:

$$
z_{\alpha}=\frac{x_{\alpha}}{2 \sqrt{\varepsilon t}}
$$

Поэтому естественно ввести новые внутренние переменные с помощью замены

$$
t=\varepsilon \theta, \quad x=\varepsilon \eta, \quad \eta=\left(\eta_{1}, \ldots, \eta_{m}\right) \in \mathbb{R}^{m} .
$$

При $\theta>0$ рассмотрим векторное поле $\mathbf{B}$, заданное как решение задачи Коши для уравнения (1) во внутренних переменных $(\theta, \eta)$

$$
\frac{\partial B_{\alpha}}{\partial \theta}+\sum_{\gamma=1}^{m} B_{\gamma} \frac{\partial B_{\alpha}}{\partial \eta_{\gamma}}=\sum_{\gamma=1}^{m} \frac{\partial^{2} B_{\alpha}}{\partial \eta_{\gamma}^{2}}
$$

с начальными условиями

$$
\left.B_{1}\right|_{\theta=0}=\left(\frac{\mu}{\varepsilon}\right)^{m} \delta(\eta) \int_{\mathbb{R}^{m}} \frac{\partial \Lambda\left(s_{1}, \ldots, s_{m}\right)}{\partial s_{1}} d s_{1} \ldots d s_{m}
$$


где $\delta(\eta)$ - это обычная дельта-функция, и при $2 \leqslant \alpha \leqslant m$

$$
\left.B_{\alpha}\right|_{\theta=0}=\left(\frac{\mu}{\varepsilon}\right)^{m-1} \Theta\left(\eta_{1}\right) \delta\left(\eta_{2}\right) \ldots \delta\left(\eta_{m}\right) \int_{\mathbb{R}^{m-1}} \frac{\partial \Lambda_{0}\left(s_{2}, \ldots, s_{m}\right)}{\partial s_{\alpha}} d s_{2} \ldots d s_{m},
$$

которые определяются из условия согласования асимптотик полей $\mathbf{H}$ и $\mathbf{B}$.

Теперь, следуя методу согласования [10], запишем составную асимптотику в главном приближении: $\mathbf{v}(x, t, \varepsilon, \mu) \approx \mathbf{W}(x, t, \varepsilon, \mu)$,

$$
\mathbf{W}(x, t, \varepsilon, \mu) \equiv \mathbf{H}\left(\frac{x}{\mu}, \frac{\varepsilon t}{\mu^{2}}\right)-\mathbf{R}\left(\frac{x}{2 \sqrt{\varepsilon t}}, \frac{\varepsilon t}{\mu^{2}}\right)+\mathbf{B}\left(\frac{x}{\varepsilon}, \frac{t}{\varepsilon}, \frac{\mu}{\varepsilon}\right),
$$

где $H_{\alpha}(\sigma, \omega)$ определяются по формуле $(7)$,

$$
\begin{aligned}
R_{1}\left(\frac{x}{2 \sqrt{\varepsilon t}}, \frac{\varepsilon t}{\mu^{2}}\right)= & \frac{\mu^{m}}{(4 \pi \varepsilon t)^{m / 2}} e^{-|x|^{2} / 4 \varepsilon t} \int_{\mathbb{R}^{m}} \frac{\partial \Lambda\left(s_{1}, \ldots, s_{m}\right)}{\partial s_{1}} d s_{1} \ldots d s_{m}, \\
R_{\alpha}\left(\frac{x}{2 \sqrt{\varepsilon t}}, \frac{\varepsilon t}{\mu^{2}}\right)= & \frac{2 \mu^{m-1}}{(4 \pi \varepsilon t)^{(m-1) / 2}} e^{-\left(x_{2}^{2}-\cdots-x_{m}^{2}\right) / 4 \varepsilon t} \int_{-x_{1} / 2 \sqrt{\varepsilon t}}^{\infty} e^{-u^{2}} d u \times \\
& \times \int_{\mathbb{R}^{m-1}} \frac{\partial \Lambda_{0}\left(s_{2}, \ldots, s_{m}\right)}{\partial s_{\alpha}} d s_{2} \ldots d s_{m}, \quad 2 \leqslant \alpha \leqslant m,
\end{aligned}
$$

$B_{\alpha}(\eta, \theta, \mu / \varepsilon)$ - решения задач $(13)-(15)$.

Обращая оператор теплопроводности в уравнении (13), получаем интегральное соотношение

$$
\mathbf{B}=\mathbf{R}-\int_{0}^{\theta} \frac{1}{\left(4 \pi\left(\theta-\theta^{\prime}\right)\right)^{m / 2}} \int_{\mathbb{R}^{m}} \exp \left[-\frac{\left|\eta-\eta^{\prime}\right|^{2}}{4\left(\theta-\theta^{\prime}\right)}\right]\left(\mathbf{B} \nabla_{\eta^{\prime}}\right) \mathbf{B} d \eta^{\prime} d \theta^{\prime},
$$

из которого с учетом условия (3) вытекает, что в правой части формулы (16) при $(x, t)$ из области $\Omega_{1}=\left\{0<t<2 \mu-|x|^{2} / \varepsilon\right\}$ разность $\mathbf{B}-\mathbf{R}$ асимптотически мала; тогда поле $\mathbf{W}(x, t, \varepsilon, \mu)$ согласно уравнениям (5) и (6) должно быть асимптотическим решением задачи. Наоборот, при $(x, t)$ из области

$$
\Omega_{2}=\left\{\mu<t+|x|^{2} / \varepsilon, t>0, t^{2}+|x|^{2}<K \varepsilon^{2}, K=\text { const }>0\right\}
$$

в выражении (16) разность $\mathbf{H}-\mathbf{R}$ асимптотически мала согласно формулам (10), $(11),(17)$ и (18); тогда поле $\mathbf{W}(x, t, \varepsilon, \mu)$ согласно уравнению (13) должно быть асимптотическим решением. Вид приведенных здесь областей в пространстве независимых переменных $(x, t)$ определяется масштабами внутренних переменных (4) и $(12)$.

Образно говоря, сингулярность, локализованная в очень малой области относительно масштабов времени порядка $\varepsilon$ и больше, может при этом являться весьма далеким будущим, если смотреть во внутренних переменных из области $\Omega_{1}$ (см. рис. 1). Для одномерного случая формула, аналогичная выражению (16), была получена в работе [13], согласно которой при $t \gg \varepsilon$ сингулярность переходит в диссипативную ударную волну.

Итак, использованный выше метод согласования позволил проследить эволючию решения уравнения Бюргерса с асимптотикой, имеющей различную структуру во 


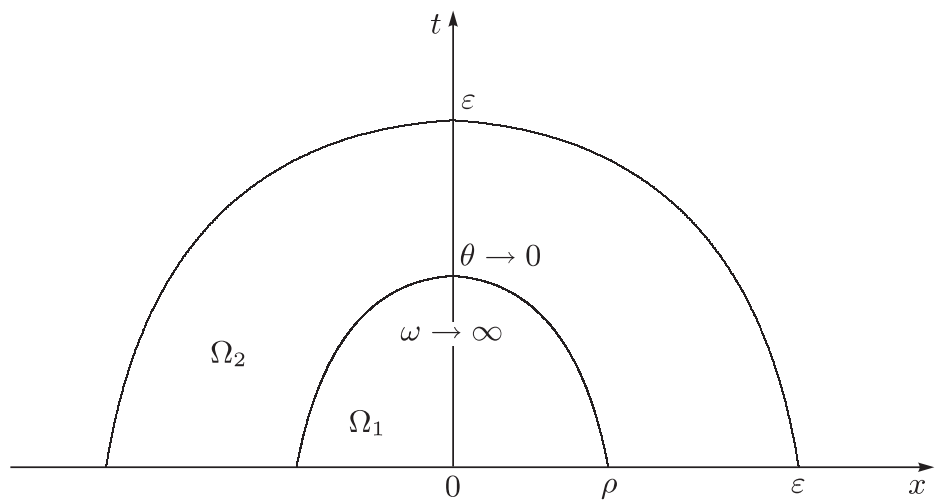

Рис. 1

вложенных друг в друга областях. Количество областей, возможно, даже увеличится при других начальных данных с особым функциональным видом асимптотики или дополнительными параметрами. В связи с этим уместно отметить, что аналогичное явление возникновения иерархии вложенных друг в друга пограничных слоев было ранее обнаружено при изучении геострофических потоков [20] и турбулентной конвекции [21]. Напротив, если в исходной постановке задачи оставить только параметр $\varepsilon$ и рассматривать разрывную начальную функцию $(\mu=0)$, то структура асимптотики вблизи сингулярности упрощается: существует уже только одна область $\Omega_{2}$ (см., например, работу [10] для одномерного случая).

Теперь выясним порядок точности, с которым поле $\mathbf{W}(x, t, \varepsilon, \mu)$ удовлетворяет уравнению Бюргерса (1) и начальному условию (2), что приводит к строгой формулировке понятия асимптотического решения в рассматриваемой задаче. В первую очередь заметим, что из формул (4), (7) и (19) следует, что при $t \rightarrow+0$ поле (16) стремится к начальным данным в правой части условия $(2)$. Далее, при $(x, t) \in \Omega_{1}$ из уравнения (6) и формул (7), (16), (19) получаем выражение

$$
\mathscr{B} \mathbf{W} \equiv \mathbf{W}_{t}+(\mathbf{W} \nabla) \mathbf{W}-\varepsilon \triangle \mathbf{W}=O\left(\frac{1}{\mu}\right), \quad \frac{\mu}{\varepsilon} \rightarrow 0, \quad \varepsilon \rightarrow 0 .
$$

Тогда

$$
\frac{\mathscr{B} \mathbf{W}}{\left|\mathbf{W}_{t}\right|+|(\mathbf{W} \nabla) \mathbf{W}|+|\varepsilon \triangle \mathbf{W}|}=O\left(\frac{\mu}{\varepsilon}\right), \quad \frac{\mu}{\varepsilon} \rightarrow 0, \quad \varepsilon \rightarrow 0
$$

поскольку в $\Omega_{1}$ знаменатель имеет порядок $\varepsilon \mu^{-2}$ согласно формулам (4) и (7). Используя уравнения (6) и (13), с учетом соотношений (17) и (18) нетрудно показать, что

$$
\mathscr{B} \mathbf{W}=((\mathbf{H}-\mathbf{R}) \nabla)(\mathbf{H}-\mathbf{R})+((\mathbf{H}-\mathbf{R}) \nabla) \mathbf{B}+(\mathbf{B} \nabla)(\mathbf{H}-\mathbf{R}) .
$$

Отсюда при $(x, t) \in \Omega_{2}$ с помощью асимптотик (10) и (11) и простых оценок производных (для всех $1 \leqslant \alpha \leqslant m$ и $1 \leqslant \beta \leqslant m$ )

$$
\frac{\partial B_{\alpha}}{\partial x_{\beta}}=O\left(\frac{1}{\varepsilon}\right), \quad \frac{\partial\left(H_{\alpha}-R_{\alpha}\right)}{\partial x_{\beta}}=O\left(\frac{\ln \left(\omega+|\sigma|^{2}\right)}{\sqrt{\varepsilon \mu}\left(\omega+|\sigma|^{2}\right)^{m / 2}}\right), \quad \omega+|\sigma|^{2}>\frac{\varepsilon}{\mu},
$$


получаем равенство

$$
\mathscr{B} \mathbf{W}=O\left(\left(\frac{\mu}{\varepsilon}\right)^{m / 2} \frac{\ln \mu}{\sqrt{\varepsilon \mu}}\right), \quad \frac{\mu}{\varepsilon} \rightarrow 0, \quad \varepsilon \rightarrow 0 .
$$

Кроме того, в $\Omega_{2}$ величина $\left|\mathbf{W}_{t}\right|+|(\mathbf{W} \nabla) \mathbf{W}|+|\varepsilon \triangle \mathbf{W}|$ имеет порядок $\varepsilon^{-1}$, что вытекает из тех же асимптотик (10), (11) и формул (17)-(19).

Таким образом, приходим к главному результату настоящей работы. Векторное поле, определенное формулой (16), является асимптотическим решением задачи (1), (2) при $t^{2}+|x|^{2}<K \varepsilon^{2}, \mu / \varepsilon \rightarrow+0 u \varepsilon \rightarrow+0$ в следуюшем смысле:

$$
\begin{gathered}
\frac{\mathbf{W}_{t}+(\mathbf{W} \nabla) \mathbf{W}-\varepsilon \Delta \mathbf{W}}{\left|\mathbf{W}_{t}\right|+|(\mathbf{W} \nabla) \mathbf{W}|+|\varepsilon \Delta \mathbf{W}|}=O\left(\left(\frac{\mu}{\varepsilon}\right)^{(m-1) / 2} \ln \mu\right), \\
\lim _{t \rightarrow+0} \mathbf{W}(x, t, \varepsilon, \mu)=\mu \nabla \Lambda\left(\frac{x_{1}}{\mu}, \ldots, \frac{x_{m}}{\mu}\right) .
\end{gathered}
$$

Следует подчеркнуть, что, поскольку в данной работе построено только главное приближение, использование стандартного определения асимптотического решения [10] через оценку значения оператора теряет смысл, даже если бы эта оценка оказалась мала. Поэтому довольно естественным было сравнение значения оператора и абсолютных величин его отдельных слагаемых, выяснив тем самым относительный порядок малости. Omnia relativa sunt.

\section{Список литературы}

[1] Дж. Уизем, Линейные и нелинейные волны, Мир, М., 1977.

[2] О. В. Руденко, С. И. Солуян, Теоретические основы нелинейной акустики, Наука, М., 1975.

[3] С. Н. Гурбатов, О. В. Руденко, А. И. Саичев, Волны и структуры в нелинейных средах без дисперсии. Приложения к нелинейной акустике, Физматлит, М., 2008.

[4] F. Bernardeau, P. Valageas, "Merging and fragmentation in the Burgers dynamics", Phys. Rev. E, 82:1 (2010), 016311, 20 pp., arXiv: 0912.3603.

[5] С.Н. Гурбатов, А.И. Саичев, С.Ф. Шандарин, "Крупномасштабная структура Вселенной. Приближение Зельдовича и модель слипания", УФН, 182:3 (2012), 233-261, arXiv: astro-ph/9311075.

[6] A. L. Melott, S.F. Shandarin, D. H. Weinberg, "A test of the adhesion approximation for gravitational clustering", Astrophys. J., 428:1 (1994), 28-34, arXiv: astro-ph/9311075.

[7] В. И. Арнольд, Особенности каустик и волновых фронтов, Фазис, М., 1996.

[8] И. А. Богаевский, "Перестройки особенностей функций минимума и бифуркации ударных волн уравнения Бюргерса с исчезающей вязкостью", Алгебра и анализ, 1:4 (1989), $1-16$.

[9] И. А. Богаевский, "Разрывные градиентные дифференциальные уравнения и траектории в вариационном исчислении", Матем. сб., 197:12 (2006), 11-42.

[10] А.М. Ильин, Согласование асимптотических разложений решений краевых задач, Наука, М., 1989.

[11] С. В. Захаров, "Сингулярные асимптотики в задаче Коши для параболического уравнения с малым параметром", Тр. ИММ УрО РАН, 21:1 (2015), 97-104.

[12] С. В. Захаров, "Особенности $A$ и $B$ типов в асимптотическом анализе решений параболического уравнения", Функи. анализ и его прил., 49:4 (2015), 82-85. 
[13] С. В. Захаров, “Задача Коши для квазилинейного параболического уравнения с большим начальным градиентом и малой вязкостью", Ж. вычисл. матем. и матем. физ., $\mathbf{5 0 : 4 ~ ( 2 0 1 0 ) , ~ 6 9 9 - 7 0 6 . ~}$

[14] T. Hollowood, J. L. Miramontes, J.S. Guillén, "Generalized integrability and two-dimensional gravitation", TMФ, 95:2 (1993), 258-275.

[15] J. Schnittger, U. Ellwanger, "Nonperturbative conditions for local Weyl invariance on a curved world sheet", TMФ, 95:2 (1993), 361-384.

[16] S. Kachru, X. Liu, M. Mulligan, "Gravity duals of Lifshitz-like fixed points", Phys. Rev. D, 78:10 (2008), 106005, 8 pp., arXiv: 0808.1725.

[17] I. Arav, S. Chapman, Y. Oz, "Lifshitz scale anomalies", JHEP, 02 (2015), 078, 58 pp., arXiv: 1410.5831.

[18] С. В. Захаров, "Задача Коши для квазилинейного параболического уравнения с двумя малыми параметрами", Докл. РАН, 422:6 (2008), 733-734.

[19] С. В. Захаров, “Асимптотическое вычисление распределения тепла на плоскости”, Тр. ИММ УрО РАН, 22, № 1, 2016, 93-99.

[20] K. Stewartson, "On almost rigid rotations. Part 2", J. Fluid Mech., 26 (1966), 131-144.

[21] F.H. Busse, "On Howard's upper bound for heat transport by turbulent convection", J. Fluid Mech., 37:3 (1969), 457-477.

Поступила в редакцию 9.10.2017, после доработки 23.01.2018 